\title{
Targeting cell motility in pulmonary arterial hypertension
}

\author{
Roxane Paulin ${ }^{1,2}$, Jolyane Meloche ${ }^{2}$, Audrey Courboulin², Caroline Lambert², \\ Alois Haromy ${ }^{1}$, Antony Courchesne ${ }^{2}$, Pierre Bonnet ${ }^{3}$, Steeve Provencher ${ }^{2}$, \\ Evangelos D. Michelakis ${ }^{1}$ and Sébastien Bonnet ${ }^{2}$

\begin{abstract}
Affiliations:
'Dept of Medicine, University of Alberta, VBRG, Edmonton, AB, and

${ }^{2}$ Dept of Medicine, Laval University, Pulmonary Hypertension Research Group CRIUPQ, Quebec, QC, Canada.

${ }^{3}$ Dept of Medecine, Tours University, Tours, France.
\end{abstract}

\section{Correspondence:}

R. Paulin, Vascular Biology Research Group, 424 HMRC University of Alberta, 116 Street and 85 Avenue, Edmonton, AB, T6G 2R3, Canada.

E-mail: rpaulindualberta.ca

ABSTRACT Pulmonary artery smooth muscle cells (PASMC), in pulmonary arterial hypertension (PAH), contribute to obliterative vascular remodelling and are characterised by enhanced proliferation, suppressed apoptosis and, a much less studied, increased migration potential. One of the major proteins that regulate cell migration is focal adhesion kinase (FAK), but its role in PAH is not fully understood. We hypothesised that targeting cell migration by FAK inhibition may be a new therapeutic strategy in PAH.

In vivo, inhalation of FAK-siRNA $(\mathrm{n}=5)$ or oral delivery of PF-228 (FAK inhibitor PF-573 228; $\mathrm{n}=5$ ) inhibited rat monocrotaline induced $\mathrm{PAH}$, improving the haemodynamics, vascular remodelling (media thickness), and right ventricular hypertrophy. In vitro, FAK was activated in PAH human lungs $(\mathrm{n}=8)$ or PASMC when compared to those form healthy subjects (Western blot, $n=5$ ), in a Src-dependent manner, as it was reversed by the specific Src inhibitor PP2. The degree of FAK phosphorylation at Y576 correlated positively with pulmonary vascular resistance in PAH patients. FAK inhibition (siRNA, PF-228 and PP2) in PAH-PASMCs induced a fivefold increase in apoptosis (percentage of terminal deoxynucleotidyl transferase dUTP nick end labelling), a 2.5-fold decrease in proliferation (\%Ki67), an 18\% decrease in cell migration (colorimetric assay) and a 50\% decrease in cell invasion (wound healing).

Suppressing PASMC migration by FAK inhibition inhibits PAH progression and may open a new therapeutic window in PAH.

@ERSpublications

Suppressing PASMC migration by FAK inhibition inhibits PAH progression and may open a new therapeutic window in PAH http://ow.ly/pRAYP

This article has supplementary material available from www.erj.ersjournals.com

For editorial comments see page 325 .

Received: Nov 102012 | Accepted after revision: May 242013 | First published online: July 112013

Support statement: This work was supported by the Heart and Stroke Foundation of Canada (HSFC, F00938310), the Canadian Institute for Health Research (CIHR, IC091477) and the Canadian Research Chair (CRC, Ic093967) to S. Bonnet. The PF-573228 was a gift from Pfizer.

Conflict of interest: Disclosures can be found alongside the online version of this article at www.erj.ersjournals.com Copyright @ERS 2014 


\section{Introduction}

Although the earliest lesion in pulmonary arterial hypertension (PAH) remains unknown, when fully expressed the disease is characterised by a proliferative and anti-apoptotic diathesis in many of the cells in small pulmonary arteries, including pulmonary artery endothelial cells (PAECs), pulmonary artery smooth muscle cells (PASMCs) and (myo)fibroblasts, as well as remodelling of the extracellular matrix. Eventually, there is obliteration of the pulmonary arterial lumen, increasing pulmonary vascular resistance, right ventricular failure and early death.

The pathogenesis of PAH is multifactorial and has fundamental similarities with cancer as PASMCs adopt a pro-proliferative, pro-survival, invasive phenotype [1-3]. In some patients, there is a genetic predisposition due to heterozygous mutations in the bone morphogenetic type II receptor (BMPR-II) leading to an impaired function of SMAD (mothers against decapentaplegic homolog) pathways [4,5] and increased p38/ MAPK (mitogen-activated protein kinase) activation [6, 7]. p53, p21, p27 and survivin are all tumour suppressor/oncogenic proteins that have also been implicated in PAH [1, 8-12]. We recently showed the critical role of the oncogenic axis c-Src (v-src sarcoma Schmidt-Ruppin A-2 viral oncogene homolog)/ STAT3 (signal transducer and activator of transcription 3)/Pim-1 (provirus integration site for Moloney murine leukaemia virus 1), accounting for increased expression and activation of the transcription factor NFAT (nuclear factor of activated T-cells) [13-15] and explaining several recognised features of PAHPASMCs including: 1) downregulation of the voltage-gated $\mathrm{K}^{+}$channel 1.5 (Kv1.5), which depolarises PASMCs, increasing intracellular $\mathrm{Ca}^{2+}\left(\left[\mathrm{Ca}^{2+}\right]_{\mathrm{i}}\right)$, and promoting proliferation); and 2) mitochondrial membrane potential $(\Delta \Psi \mathrm{m})$ hyperpolarisation and inhibition of mitochondria-derived reactive oxygen species (mROS) generation, both of which suppress apoptosis [16]. We also demonstrated that STAT3 sustains its own activation through a positive feedback loop involving Src and the microRNA miR-204 [14].

Focal adhesion kinase (FAK) and Src are cytoplasmic non-receptor tyrosine kinases that have been implicated in cancer. FAK and Src both integrate signals coming from several signalling pathways, including the extracellular matrix (ECM)/integrins [17], growth factors [18, 19], G-protein coupled receptor (GPCR) [20] and signals from the cytoskeleton [21, 22]. These upstream signals lead to FAK and Src autophosphorylation (on Y397 and 418, respectively) and subsequent formation of a complex, promoting Src-dependent FAK activation by phosphorylation on additional sites [23, 24]. Hence, FAK functions as a signalling hub and is involved, to some degree, in most of the signal transduction processes that orchestrate cell differentiation, growth, survival, adhesion, invasion and migration, involving several pathways like STATs (signal transducers and activators of transcription) [25], MAPK, Rho GTPase/RhoA [26] or paxillin [27].

FAK upstream and downstream signals have been found to be activated in PAH [28], and the implication of migration processes in the pathogenesis of the disease has been suggested, but the exact role of FAK and the effects of its inhibition in PAH have not been adequately studied. FAK inhibition in cancer has shown promise in terms of effectiveness and toxicity in early phase trials [29]. Thus, we hypothesised that targeting migration by FAK inhibition would be beneficial in PAH as well.

\section{Materials and methods}

All the experiments were performed with the approval of the Laval University Ethic and Biosafety Committee. The investigation conforms to the Guide for the Care and Use of Laboratory Animals published by the US National Institutes of Health [30] and the principles outlined in the Declaration of Helsinki [31].

\section{Cell culture}

PAH-PASMCs were isolated from $<1500 \mu \mathrm{m}$ in diameter small pulmonary arteries from two males with idiopathic PAH (iPAH) (aged 31 and 48 years) from lung transplant [1]. Both patients had right catheterisation that confirmed pulmonary hypertension (mean pulmonary arterial pressure (PAP) $>25 \mathrm{mmHg}$ at rest). Five control PASMC cell lines (three males aged 45, 21, and 64 years and two females aged 17 and 35 years) were purchased (Cell Application group, San Diego, CA, USA). All PASMC cell lines were grown in high-glucose DMEM media supplemented with 10\% FBS (Gibco, Invitrogen, Burlington, ON, Canada) and 1\% antibiotic/antimytotic (Gibco, Invitrogen, Burlington, ON, Canada) [32]. All cells were used until the fifth passage.

\section{Cell treatments}

siRNA (from AMBION, Austin, TX, USA) were transfected at $20 \mathrm{nmol} \cdot \mathrm{L}^{-1}$ final concentration with calcium chloride [13]. After $24 \mathrm{~h}$, the medium was changed and experiments were performed $48 \mathrm{~h}$ after the beginning of the transfection. PP2 and PP3 were dissolved in DMSO and applied, for $48 \mathrm{~h}$, at a final concentration of $10 \mu \mathrm{M}$. PF-573228 (PF-228), an FAK inhibitor (a gift from Pfizer, Pointe-Claire/Dorval, QC, Canada) as a gift as was dissolved in methanol and a final concentration of $10 \mu \mathrm{M}$ was applied for $48 \mathrm{~h}$. 
In vivo experiments

250-350 g Sprague-Dawley rats were injected subcutaneously with $60 \mathrm{mg} \cdot \mathrm{kg}^{-1}$ of monocrotaline (MCT) $(\mathrm{n}=20)$. Intratracheal nebulisation of $1 \mathrm{nmol}$ siSCR (Ambion, Austin, TX, $)(\mathrm{n}=5)$ and $1 \mathrm{nmol}$ siFAK (Ambion) $(\mathrm{n}=5)$ were given on day 18, once pulmonary hypertension was established as previously described [13]. Freshly prepared PF-228 was administered by gavage $(n=5)$ twice a day at a concentration of $30 \mathrm{mg} \cdot \mathrm{kg}^{-1}$ per day.

\section{Statistics}

Data are presented as mean \pm SEM. For comparison between two means we used a paired t-test. For comparison between more than two means we used a one-way ANOVA followed by a Tukey post hoc test. Further details can found in the online supplementary material.

\section{FAK inhibition in vivo inhibits MCT-induced PAH}

Rats were injected, subcutaneously, with $60 \mathrm{mg} \cdot \mathrm{kg}^{-1} \mathrm{MCT}-\mathrm{PAH}$ and disease progression and exercise capacity were followed noninvasively with Doppler echocardiography and standard treadmill tests. After $\mathrm{PAH}$ was established (decreased pulmonary artery acceleration time (PAAT) at day 18, as previously described [13]), FAK versus scramble siRNAs $(1 \mathrm{nmol})$ were selectively delivered once into the lung by intratracheal nebulisation [16]. PF-228 was administered by gavage twice a day $\left(30 \mathrm{mg} \cdot \mathrm{kg}^{-1}\right.$ per day). Two weeks later, rats were anesthetized and PA pressures were measured in close-chest rats, using a telemetry catheter inserted in the right jugular vein.

FAK inhibition was associated with lower PAP $(20 \pm 1 \mathrm{mmHg}, \mathrm{n}=5 ; \mathrm{p}<0.05)$ compared to siSCR treated rats $(40 \pm 1.5 \mathrm{mmHg}, \mathrm{n}=5 ; \mathrm{p}<0.05)$ (fig. 1a). Pressures were also lower after treatment with $\mathrm{PF}-228$, $(25 \pm 1.5 \mathrm{mmHg}, \mathrm{n}=5 ; \mathrm{p}<0.05)$. Hearts were harvested and right ventricle (RV) hypertrophy (RV weight/ left ventricle $(\mathrm{LV})$ + septum weight ratio) was decreased by $20 \%$ in PF-228 and siFAK-treated rats, compared to vehicle (water) and siSCR-treated rats, respectively, $(\mathrm{n}=5$ in each group; $\mathrm{p}<0.05)$ (fig. 1b). The same effect on RV hypertrophy was observed with the two therapeutic interventions despite a different effect on the level of PAP.

This may be due to the fact that on the one hand PF-228 was administered systemically and may have some direct effects on RV, while on the other hand the siFAK was administered in a more specific manner, perhaps achieving more robust effects on the pulmonary vasculature, while affecting RV hypertrophy only indirectly.

In order to measure the distal extension of pulmonary arterial muscularisation in vivo, we measured the percentage of muscularisation in arteries $<50 \mu \mathrm{m}$ (fig. 1c). FAK inhibition by siRNA or PF-228 reduced the percentage of fully muscularised arteries and increased the percentage of nonmuscularised arteries in comparison to MCT-PAH+siSCR rats or vehicle.

By following the progression of the disease noninvasively with treadmill test and echocardiography during the 2 weeks of treatment (fig. 1d-f), we determined that the therapeutic intervention completely inhibited the progression of the disease, stopping the decrease in PAAT values and the increase in RV hypotrophy, which progressed in the MCT-PAH groups, while it did not reverse the disease or normalised the measured parameters.

FAK is activated in PAH-PASMCs and its full kinase activation is dependent on Src kinase activity.

In order to study more in depth the role of FAK in PAH pathogenesis, we performed in vitro studies with cultured PASMCs isolated from control and PAH patients. We studied FAK's phosphorylated state, using immunostaining and Western blots, and we focused on two important phosphorylation sites that are critical for the function of FAK: 1) FAK phosphorylation on residue tyrosine 397 (PY397-FAK), a known result of FAK autophosphorylation and a facilitator of FAK's interaction with Src [24, 25]; and 2) PY576-FAK, which is located within the kinase domain and responsible for full kinase activity [33]. Both PY397-FAK and PY576-FAK residues were phosphorylated with a twofold increase in PAH-PASMCs compared to controlPASMCs (fig. 2). Interestingly, PY397 appeared to be cytosolic, while PY576 was preferentially located in nuclei (online supplementary material fig. S1). To verify that Y576 phosphorylation of FAK depends on Src kinase activity, we treated PAH-PASMCs with the Src inhibitor PP2 or its inactive analogue PP3. Effective Src inhibition by PP2 (i.e. decreased phophorylation on tyrosine 418, PY418-Src) was associated with decreased PY576-FAK but not PY397-FAK, compared to PP3, thereby confirming that Y576 phosphorylation depends on Src activation, while PY397-FAK does not (fig. 2).

FAK has been described to promote an open and active Src conformation, resulting in a sustained Src activation. In order to study if FAK plays a role in Src activation in PAH, we used a silencer RNA directed against FAK (siFAK $20 \mathrm{nM}$ ) and the pharmacological FAK inhibitor PF-228 (10 $\mu \mathrm{M})$ [34]. FAK inhibition 

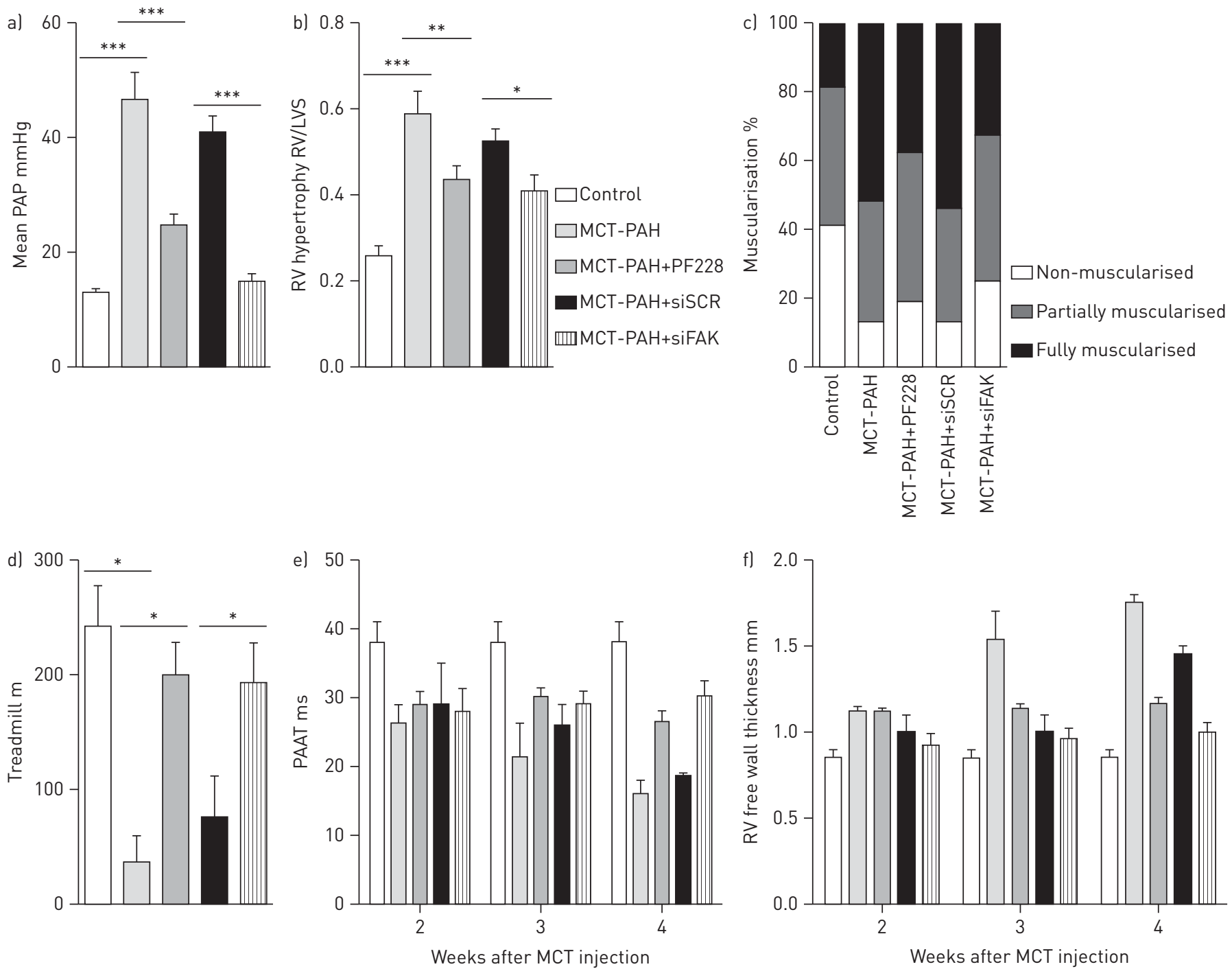

FIGURE 1 Focal adhesion kinase (FAK) inhibition by intratracheal nebulisation of siRNA or gavage with PF-228 (FAK inhibitor PF-573228) reverses monocrotaline pulmonary arterial hypertension (MCT-PAH). Intratracheal nebulisation of $1 \mathrm{nmol}$ siSCR $(\mathrm{n}=5)$ and $1 \mathrm{nmol}$ siFAK $(\mathrm{n}=5)$ were given on day 18 , once pulmonary hypertension had been established. Freshly prepared PF-228 was administered by gavage $(\mathrm{n}=5)$ twice a day at the concentration of $30 \mathrm{mg} \cdot \mathrm{kg}^{-1}$ per day. a) Invasively, mean pulmonary arterial pressure (PAP) ( $n=5$ per group) measured by right catherisation in closed chest rats, were decreased by FAK inhibition compared to MCT-PAH and MCT-PAH+siSCR. b) Right ventricle (RV) hypertrophy ( $\mathrm{n}=5$ per group), measured by RV weight/left ventricle (LV)+septum weight ratio, were also significantly improved by FAK inhibition. c) Moreover, in keeping with the inhibition of cell motility associated with FAK inhibition, the percentage of fully muscularised arteries was decreased while the percentage of nonmuscularised arteries was increased with FAK inhibition compared to MCT-PAH or MCT-PAH+siSCR. The effects of FAK inhibition by either siFAK or PF-228 were studied, noninvasively, by d) exercise capacity on a treadmill ( $\mathrm{n}=5$ group) and echocardiography Doppler, and FAK inhibition improved the distance travelled on the treadmill; increase the e) pulmonary acceleration time; and decrease f) the RV free-wall thickness. ${ }^{*}: \mathrm{p}<0.05 ;{ }^{* *}: \mathrm{p}<0.01{ }^{* * *}: \mathrm{p}<0.001$.

by both siRNA and PF-228 reduced Src phosphorylation on Y418, suggesting that the cooperation between the two partners is a part of the process resulting in signal transduction in PAH (fig. 2 and online supplemental fig.1b).

We then studied, by immunostaining, the state of FAK phosphorylation on the Y576/577 (PY576-FAK) on lung sections from four control patients and four PAH patients (online supplemental table S1). We found a twofold increase in PY576-FAK in PAH-patients compared to controls (online supplemental fig.2). Moreover, the increase in PY576-FAK paralleled the increase in pulmonary vascular resistance in the patients with $\mathrm{PAH}$, implying a positive correlation with disease progression.

Similar to the experiments with human tissues, in our rat model of PAH, we used both immunostaining and immunoblots and showed that FAK was aberrantly activated with a threefold increase in PY397 and a fourfold increase in PY576, compared to control rats (supplemental figs 3 and 4). Our therapeutic 
a)

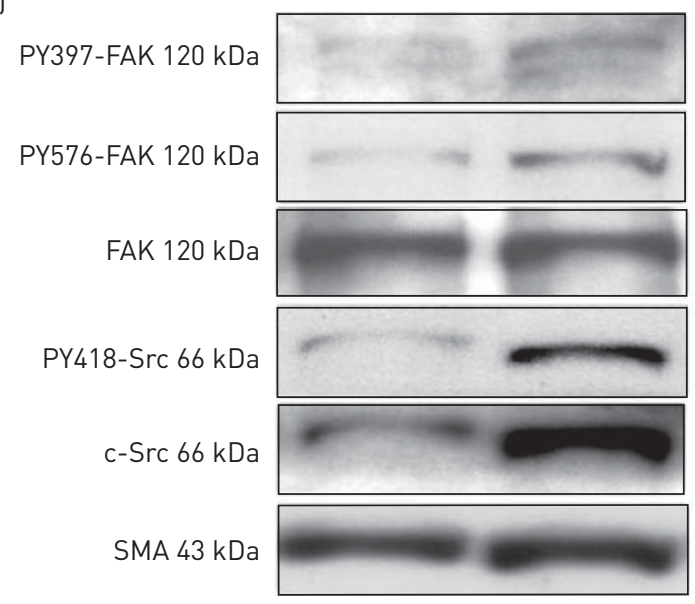

b)

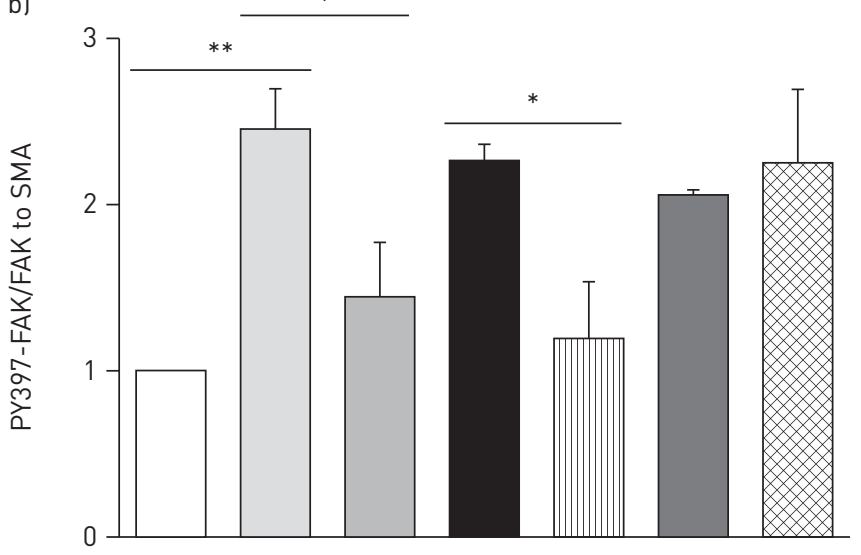

c)

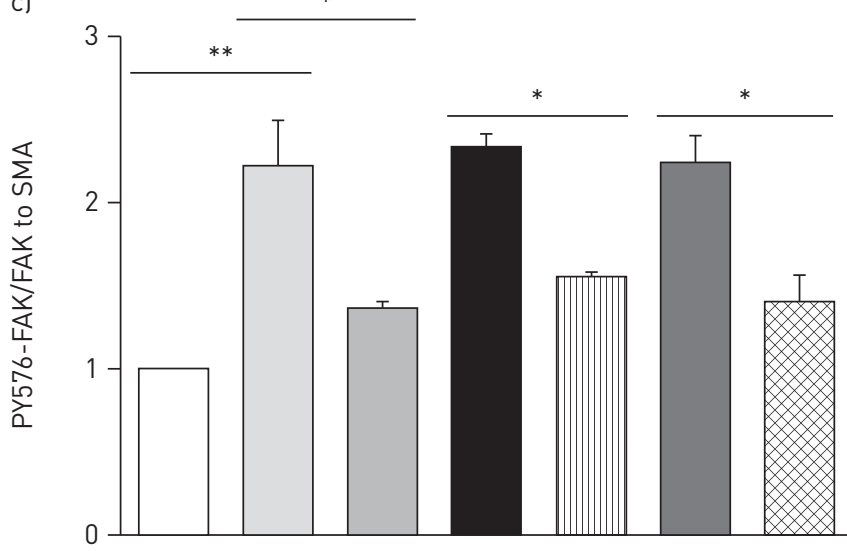

d

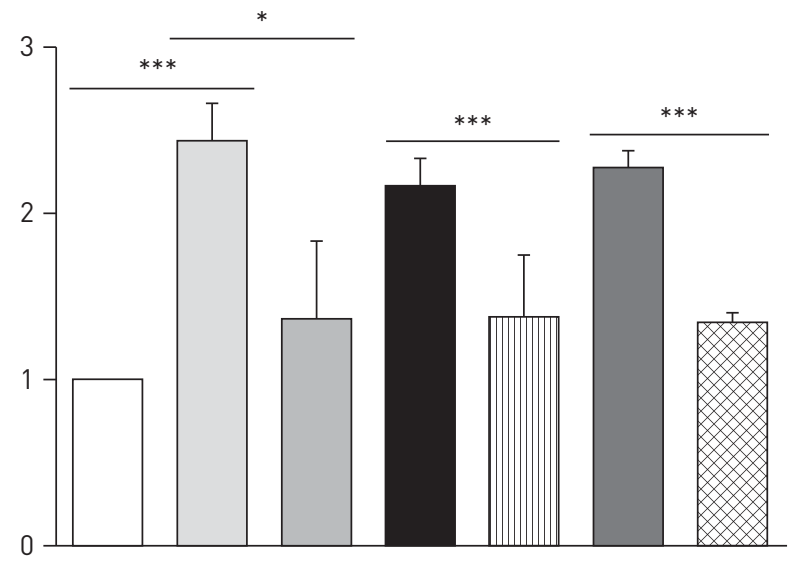

+ SiSCR

+ SiFAK

$+\mathrm{PF}-228$
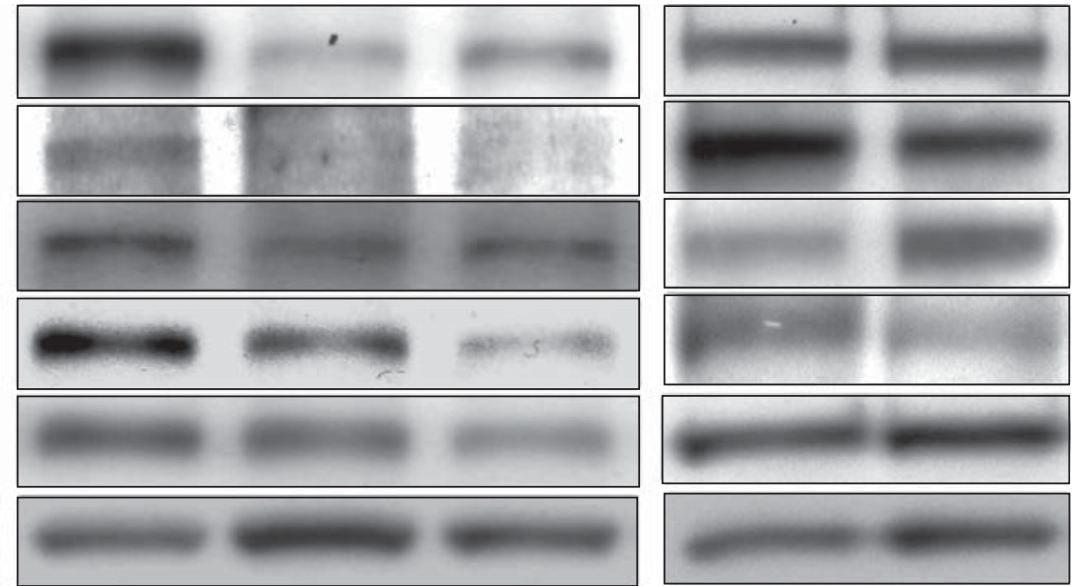

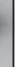



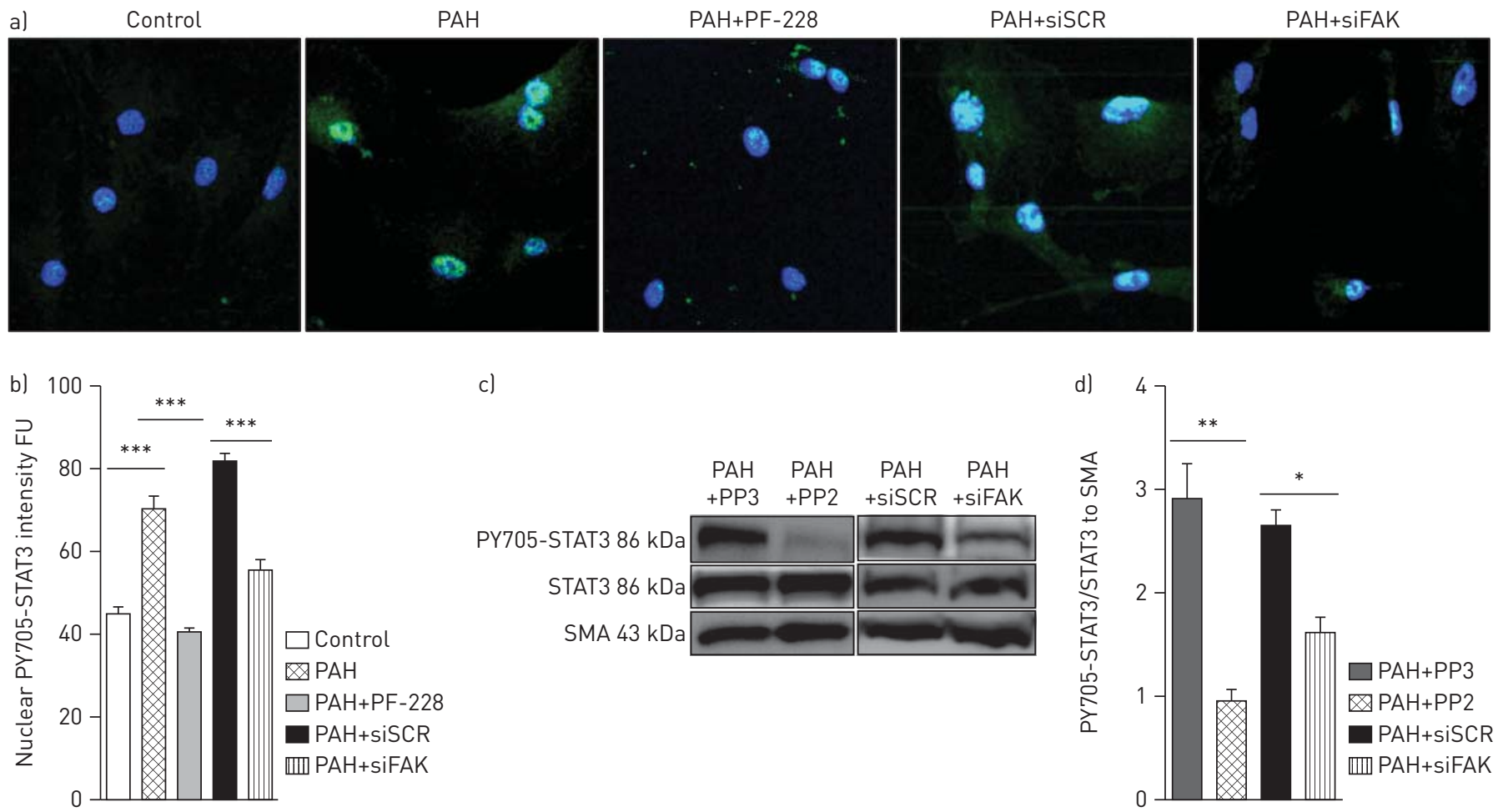

FIGURE 3 Focal adhesion kinase (FAK) inhibition decreases signal transducer and activator of transcription (STAT) 3 activation in pulmonary arterial hypertension (PAH) pulmonary artery smooth muscle cells (PASMCs). a) STAT3 activation was evaluated by nuclear translocation assay measuring the amount of P-STAT3 in green in the nucleus, which was stained blue with 4,6-diamidino-2-phenylindolein. STAT3 is activated in PAH-PASMCs compared to control cells. FAK inhibition by either siRNA or the compound PF-228 (FAK inhibitor PF-573 228) decreases STAT3 activation in PAH-PASMCs. The influence of FAK inhibition on STAT3 activation has been further studied by Western blot (c). FAK inhibition by siRNA is associated with 50\% decrease in STAT3 PY705 while Src inhibition by PP2 is associated by $75 \%$ decrease in STAT3 activation (d). ${ }^{*}: \mathrm{p}<0.05 ;{ }^{* *}: \mathrm{p}<0.01 ;{ }^{* * *}: \mathrm{p}<0.001$.

interventions in this model, both PF-228 gavage and siFAK intratracheal nebulisation, were able to significantly decrease this inappropriate FAK activation (supplemental figs 3 and 4).

\section{FAK inhibition decreases STAT3 activation in PAH-PASMCS}

We previously described that Src activation is associated with activation and nuclear translocation of the transcription factor STAT3 (i.e. increased phosphorylation on tyrosine 705, PY705-STAT3). In order to study whether FAK is implicated in signal transduction through STAT3, we measured the level of nuclear PY705-STAT3 in PASMCs-PAH treated with PF228 and siFAK. Both PF-228 and siFAK decreased nuclear PY705-STAT3 levels compared to vehicle-treated and scrambled RNA-treated PAH-PASMCs (fig. 3a), a decrease that approaches the level seen in the normal control. We also found that STAT3 activation (level of PY705-STAT3 by Western blot and nuclear translocation by immunofluorescence) was decreased in distal PAs after FAK inhibition in vivo with siFAK or PF-228 (supplemental fig.3).

\section{FAK inhibition decreases the PAH-PASMCs migratory potential}

We then studied the effect of FAK inhibition on PASMC motility. PASMCs migration was studied using a modified Boyden chamber as described in the methods section. PAH-PASMCs had increased motility levels $(\mathrm{OD}=2.75 \pm 0.3$, fig. $4 \mathrm{a})$ compared to control-PASMCs $(\mathrm{OD}=2.1 \pm 0.7$, fig. 4a). FAK inhibition by siRNA in PAH-PASMCs caused a $18 \%$ decrease in migration (OD $2.2 \pm 0.7$ versus $2.7 \pm 1.2$ for scrambletransfected cells, fig. 4a). Similar results were obtained with PF228. These results show directly that FAK is important in the enhancement of PASMCs motility in PAH.

\section{FAK and STAT3 inhibition decreases wound healing response in PAH-PASMCS}

We then investigated the effect of FAK inhibition on PASMCs invasion by standard in vitro wound healing assays (fig. 4b). As STAT3 has been previously implicated in smooth muscle cells invasion [35, 36], we also performed this experiment in the presence of STAT3 silencer RNA.

After $24 \mathrm{~h}$, PAH-PASMCs exhibited a better response along the wounded edge margin than the control PASMCs. PAH-PASMCs migrated into the wounded area and completely filled it, as shown by the fact that 

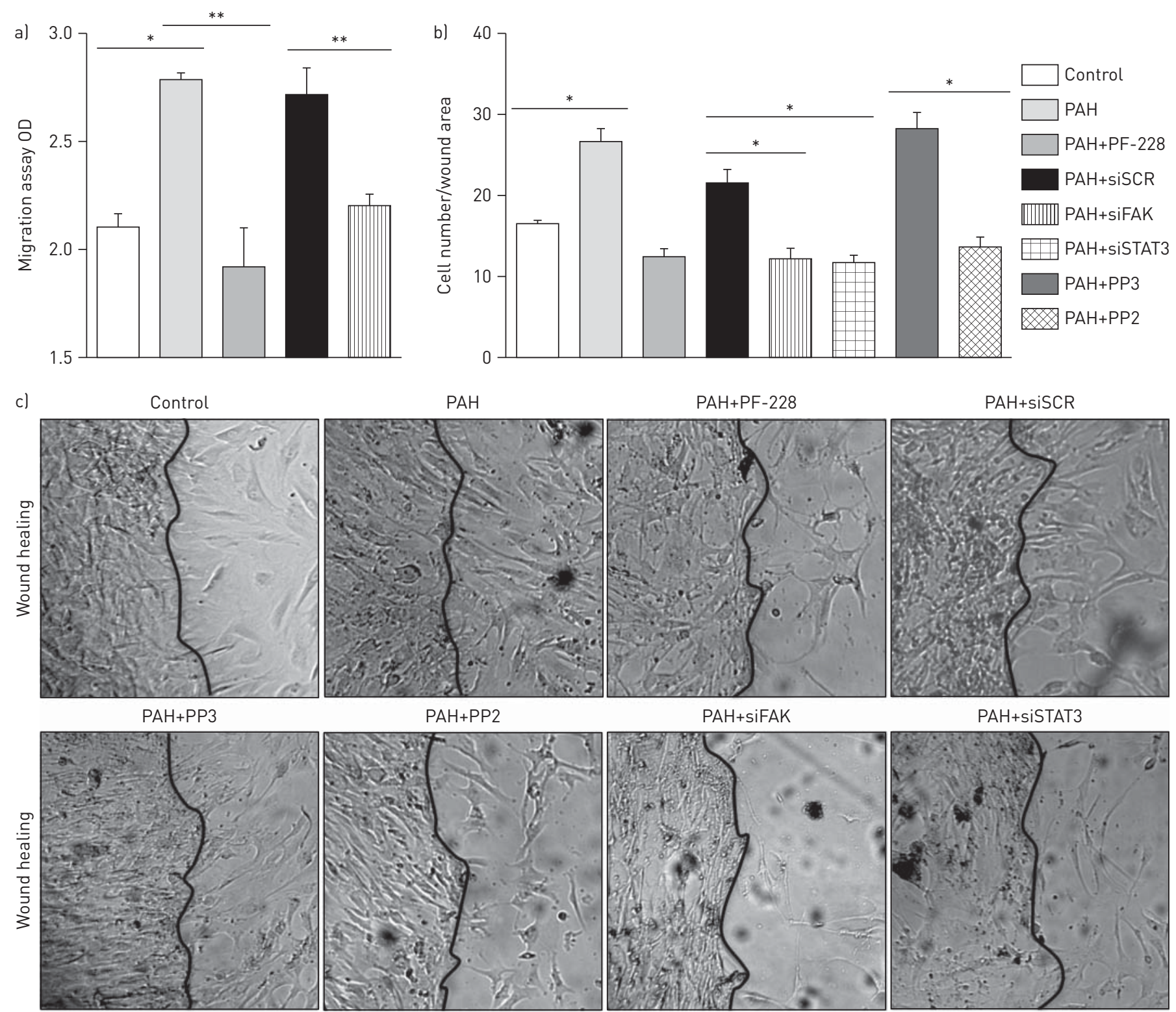

FIGURE 4 a) Focal adhesion kinase (FAK) inhibition decreases pulmonary arterial hypertension pulmonary artery smooth muscle cells (PAH-PASMCs) motility. PASMCs motility was measured using a modified Boyden chamber experiments and $10 \%$ serum media as chemoattractant in the lower chamber. Migrating cells were stained, the stain was extracted and optical density (OD) was measured at $560 \mathrm{~nm}$. FAK inhibition in PAH-PASMCs is associated with decreased migration $\left(\mathrm{n}=3\right.$, experiments in duplicate). ${ }^{*}: \mathrm{p}<0.05 ;{ }^{* *}: \mathrm{p}<0.01$. b) FAK inhibition decreases PAH-PASMCs invasion. In vitro wound healing assays were also performed in PAH-PASMCs with FAK inhibition. Cell motility/invasion was assessed by the number of cells that invaded the wound divided by the wound area. FAK inhibition (siRNA, PF-228 (FAK inhibitor PF-573 228) and Src-dependent inhibition by PP2) as well as signal transducer and activator of transcription (STAT) 3 inhibition (siRNA) decreases the PAH-PASMCs pathological response to wound healing $(n=3) .^{*}: p<0.05 ;{ }^{* *}: p<0.01$.

the number of cells in the wound area increased by almost twice as much when compared with the controlPASMCs (fig. 4b). PAH-PASMCs transfected with FAK-siRNA exhibited only a limited response along the wound edge margin and did not completely repopulate the open space compared to the scrambled treated cells (fig. 4b). Similar results were obtained with wound-healing assays using PF-228 and PP2 (Srcdependent FAK inhibition) when compared to PAH-PASMCs and PP3, respectively. These results demonstrate the critical role of the signalling hub FAK in the enhanced wound-healing response seen in PAH-PASMCs. They also show that FAK inhibition is effective in restoring a normal wound-healing response, decreasing invasion by $50 \%$ and at a level similar to that of the control cells. Interestingly, STAT3 also decreased PAH-PASMC motility, suggesting again a strong cooperation between STAT3 and Src/FAK. 
PY576-FAK is localised in focal adhesion point and in the nucleus, and is associated with actin reorganisation

The ability of Src/FAK/STAT3 to decrease the aberrant motility of PAH-PASMCs was further studied using immunofluorescence. FAK regulates focal adhesion turnover required for efficient cell mobility [37] by enhancing paxillin phosphorylation and subsequent actin fiber (F-actin) formation [38]. F-actin reorganisation was imaged using the Texas Red-phalloidin dye (Invitrogen, Burlington, ON, Canada) and antibodies against Paxillin were used to image focal adhesion points. We also stained with an antibody against PY576-FAK in order to identify its localization during cell motility. PAH-PASMCs were characterized by reorganization of the actin cytoskeleton and formation of lamellipodia, whereas control PASMCs only showed small actin protrusions (fig. 5). PY576-FAK was increased in PAH-PASMCs, and although it was present at focal adhesion points, it exhibited high levels in the nucleus as well. FAK inhibition by siRNA or PF228, and Src inhibition by PP2, largely abolished actin filament assembly and formation of typical focal adhesion plaques (as shown by Paxillin expression and lamelipodium formation), although small actin protrusions were still observed. Nuclear intensity of PY576-FAK was decreased by siFAK, PF228, siSTAT3 and PP2 compared to appropriate controls siSCR and PP3, confirming a strong cooperation between Src, STAT3 and FAK. Nonetheless, the exact function of nuclear PY576-FAK in PAH is still unknown.

\section{FAK inhibition decreases PAH-PASMCs proliferation and resistance to apoptosis}

Since FAK activation was previously associated with increased proliferation and resistance to apoptosis in SMCs $[39,40]$, we studied the ability of FAK inhibition to reverse the PASMCs hyperproliferative and apoptosis-resistant phenotype. PASMCs were grown in media supplemented with $10 \%$ fetal bovine serum (FBS), a condition known to promote proliferation [16, 32]. We found that FAK inhibition by either siRNA, PF-228 or a PP2-dependent Src inhibition in PAH-PASMCs decreased by 2.5 -fold the number of Ki67 positive cells and by twofold the number of proliferating cell nuclear antigen-positive cells (PCNA) (fig. 6a and b) compared to controls, normalising PAH-PASMCs proliferation rates.

To study the effect of FAK inhibition on the mitochondrial membrane potential $(\Delta \Psi \mathrm{m})$ (an index of the threshold for mitochondrial-dependent apoptosis), we used tetramethylrhodamine methyl-ester (TMRM) imaging in live PAH-PASMCs transfected with FAK siRNA, siSCR or treated with PF-228, PP2 or its inactive analogue PP3. FAK inhibition caused a significant $\Delta \Psi \mathrm{m}$ depolarisation (fig. $6 \mathrm{c}$ and $\mathrm{d}$, and online supplement fig. 6). In keeping with these data, FAK inhibition by siRNA in serum-starved PAH-PASMCs $(0.1 \%$ FBS $)$ induced a fivefold increase in apoptosis, while PF228 or PP2 induced a fourfold increase (fig. $6 \mathrm{c}$ and $\mathrm{d})$.

FAK inhibition in vivo is associated with decreased PASMCs proliferation and increased apoptosis in distal pulmonary arteries, leading to decreased vascular remodelling

FAK inhibition (with siFAK or PF-228) in vivo was associated with increased apoptosis (terminal deoxynucleotidyl transferase dUTP nick end labelling (TUNEL)) and decreased proliferation (Ki67 expression) of smooth muscle actin-positive cells in resistance pulmonary arteries (PAs) (fig. 7a). This was associated with a reversal of media hypertrophy in distal PAs (fig. 7a and d). These therapies did not appear to decrease proliferation and apoptosis in vW-Factor positive cells (Supplemental Fig. 7), suggesting that their effects may be specific to PASMC. Taken together with the effects of these therapies on muscularization of the PAs in the same animals (fig. 1c) these data show that FAK inhibition can inhibit vascular remodelling in $\mathrm{PAH}$ by a number of mechanisms including effects on proliferation, apoptosis and cell motility.

\section{Discussion}

Here we demonstrated for the first time that FAK plays a critical role in $\mathrm{PAH}$ pathogenesis by regulating proliferation, resistance to apoptosis, migration and invasion of PASMC, all critical determinants of the obliterative vascular remodelling that characterises this disease. FAK phosphorylation on Y397 has been described in PAH, as a consequence of an increased expression of integrin [41]. Here we show that FAK is also phosphorylated on Y576, in a Src-dependent manner, thus allowing activation of many other signalling pathways, including STAT3. We also found that STAT3 phosphorylation is decreased by FAK inhibition and that STAT3 activation participates in PAH-PASMCs migration and wound healing. While STAT3 role in cell motility were described several times in cancer, it is the first time that this has been shown in vascular smooth muscle cells.

STAT3 is known to regulate cell motility through its transcriptional activating functions [42], but there is also evidence that STAT3 (non-tyrosine-phosphorylated form) mediates cell migration by regulating microtubule polymerisation [43-45]. In addition, phosphorylated STAT3 has also been found to localize 
a)

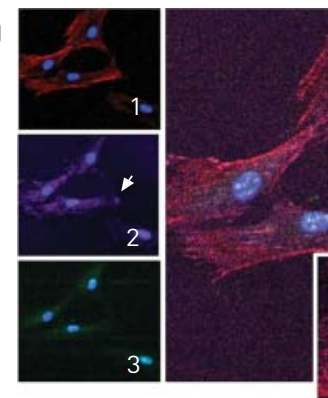

Control

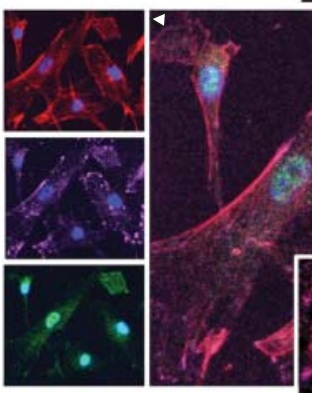

$\mathrm{PAH}+\mathrm{SiSCR}$



$\mathrm{PAH}+\mathrm{PP} 3$
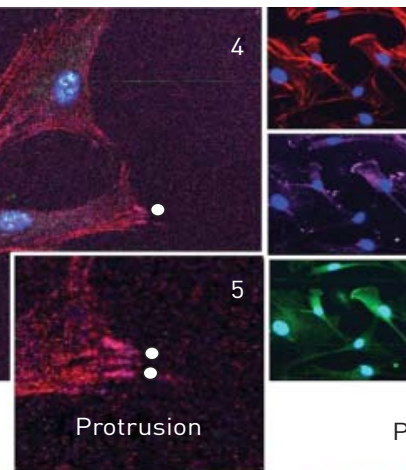

$\mathrm{PAH}$

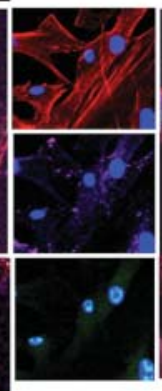

$\mathrm{PAH}+\mathrm{SIFAK}$
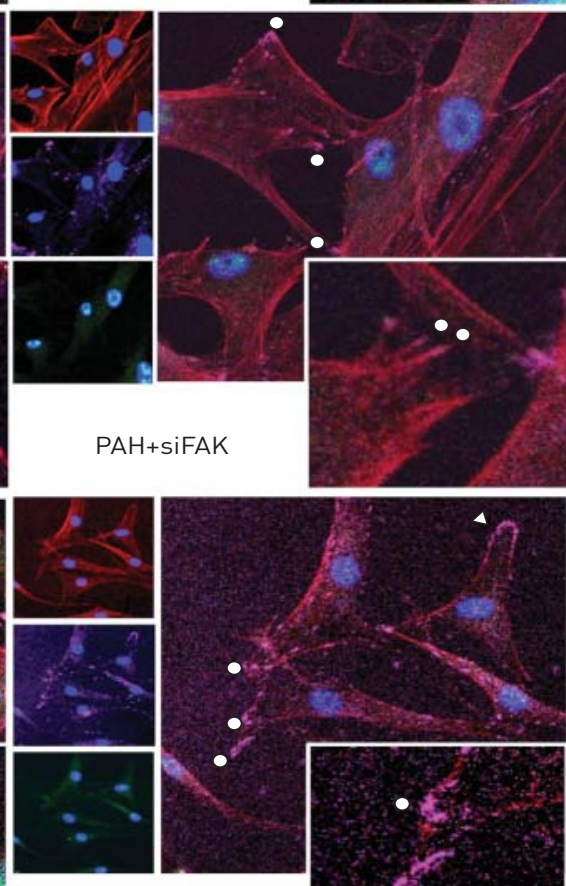

$\mathrm{PAH}+\mathrm{PP} 2$

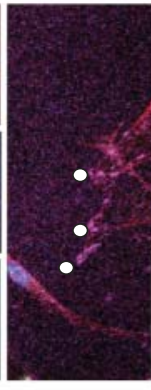

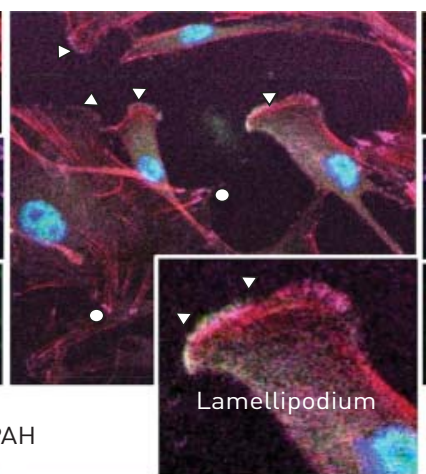
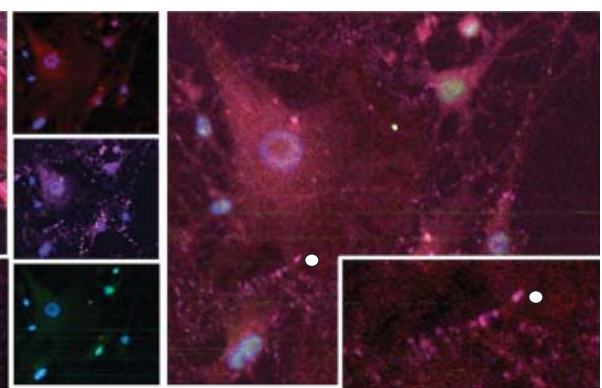

$\mathrm{PAH}+\mathrm{PF}-228$
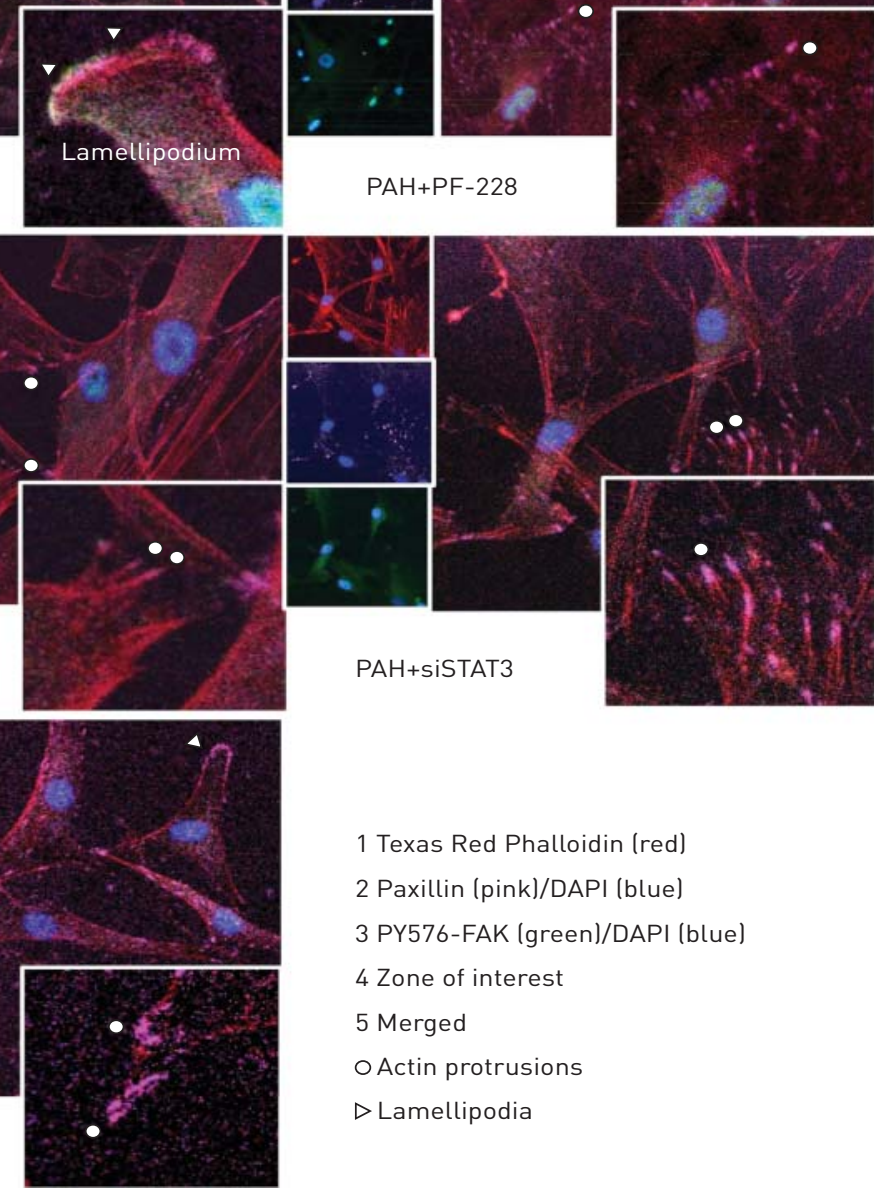

1 Texas Red Phalloidin (red)

2 Paxillin (pink)/DAPI (blue)

3 PY576-FAK (green)/DAPI (blue)

4 Zone of interest

5 Merged

OActin protrusions

$\triangleright$ Lamellipodia
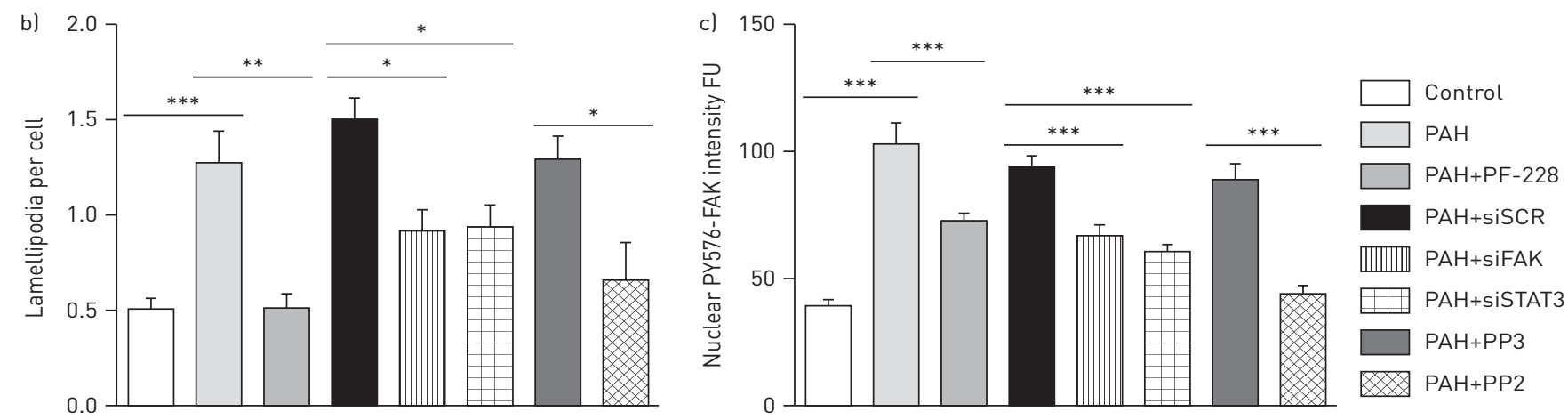

FIGURE 5 Focal adhesion kinase (FAK) inhibition decreases pulmonary arterial hypertension (PAH) pulmonary artery smooth muscle cells (PASMCs) actin reorganisation. F-actin re-organisation was stained using Texas Red Phalloidin (1) and focal adhesion dots were visualised using Paxillin (2) staining in far red (pink). PY576-FAK (3) was stained in green and nucleus by 4,6-diamidino-2-phenylindolein in blue. Representative pictures of typical structures observed in each group are presented (4 and 5). As PY576-FAK is present in the nucleus, nuclear PY576-FAK intensity mean data are presented in c). PAH-PASMCs are characterised by the re-organisation of the actin cytoskeleton with the formation of motile structures, such as lamellipodia (white arrowheads, 1.5 lamellipodia per cell versus 0.5 in controls) whereas control PASMCs only arbor small actin protrusions (white circles). FAK, Src and signal transducer and activator of transcription (STAT) 3 inhibition decreased the presence of lamelipodia in PAH-PASMCs and decreased the amount of nuclear PY576-FAK. ${ }^{\star}: \mathrm{p}<0.05$; ${ }^{* *}: \mathrm{p}<0.01 ;{ }^{* *}: \mathrm{p}<0.001$. 

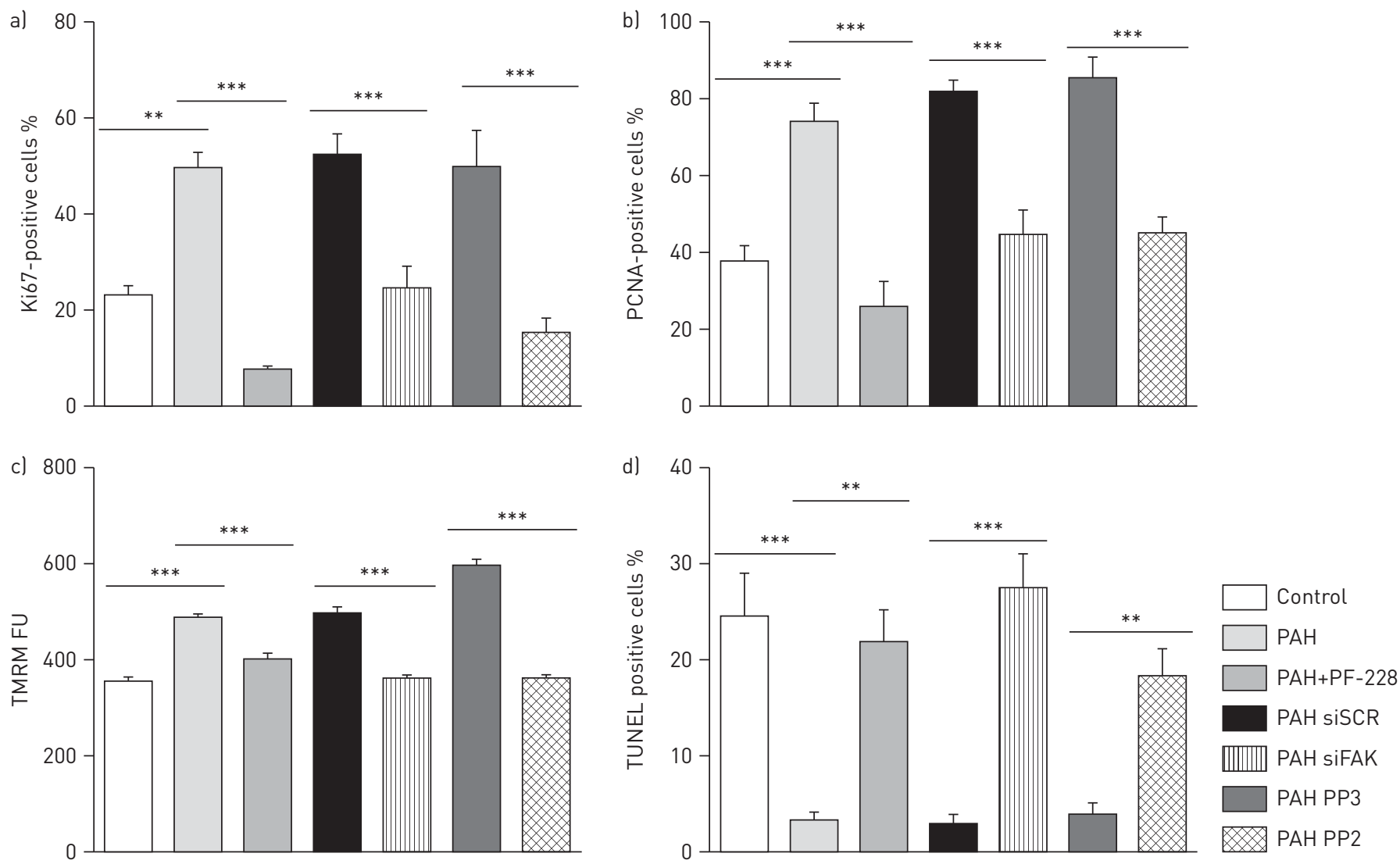

FIGURE 6 a, b) Focal adhesion kinase (FAK) inhibition decreases pulmonary arterial hypertension (PAH) pulmonary artery smooth muscle cells (PASMCs) proliferation. PAH-PASMCs had increased proliferation rates measured by the percentage of proliferating cell nuclear antigen and Ki67-positive cells, compared to healthy PASMCs. Both FAK siRNA and PP2 significantly decreased PAH-PASMC proliferation compared to the appropriate controls siSCR and PP3 ( $\mathrm{n}=100-$ 150 cells by experiment, in at least three experiments by cell line in five controls and the PAH-patient's PASMCs). ${ }^{* *}: \mathrm{p}<0.01 ;{ }^{* * *}: \mathrm{p}<0.001 . \mathrm{c}$ and $\left.\mathrm{d}\right)$ FAK inhibition increases PAH-PASMCs mitochondrial dependent apoptosis. FAK inhibition by both siRNA and PP2 in PAH-PASMC reverses $\triangle \Psi m$ hyperpolarisation (TMRM) and decreases apoptosis (percentage terminal deoxynucleotidyl transferase dUTP nick end labelling (TUNEL)) compared to siSCR and PP3.

within focal adhesions, interacting with focal adhesion proteins and contributing to ovarian cancer cell motility [46].

We were particularly interested in PY576-FAK in this study as it reflects maximal FAK kinase activity [33]. Phosphorylation of this residue is particularly enhanced by laminar shear stress [47]. This is consistent with our model, in which the increase in pressure creates cyclic stretch and shear stress on the vessel wall $[48,49]$. Moreover, FAK activation seems to be particularly enhanced by mechanical stress as Src/FAK axis activation has been associated with a $30 \mathrm{mmHg}$ increase in systemic pressure [50]. This suggests that the increase in pressure in PAH might be implicated in the maintenance and amplification of FAK activation, leading to the worsening of PAH.

Interestingly, we found that PY576-FAK is also localised in the nucleus, while PY397-FAK is mostly cytoplasmic. It has been demonstrated that p53 regulation, which is protective against PAH [8] and downregulated in MCT-PAH [12], is dependent on FAK nuclear translocation. Indeed, FAK inactivates p53 in a kinase-independent manner via its FERM domain ( $\mathrm{F}$ for 4.1 protein, $\mathrm{E}$ for ezrin, $\mathrm{R}$ for radixin and $\mathrm{M}$ for moesin), acting as a scaffold protein to enhance Mdm2-dependent p53 ubiquitination and degradation [51-54].

Here we focused on PASMCs as the less studied cell motility was our primary endpoint. However, there is evidence to suggest that FAK may also be activated in PAH pulmonary artery endothelial cells (PAEC). For example, in PAH-PAEC there is increased STAT3-dependent proliferation, increased migration, and decreased apoptosis compared with cells derived from healthy control lungs [55], much like in PASMC. STAT3 hyperactivation has also been found in PAECs following MCT injection in rats, leading to PAECs proliferation [56]. These data are in keeping with a potential activation of FAK in PAEC. Indeed, in a different PAH model (SU5416+hypoxia) Copper restriction (an approach that has been shown to inhibit FAK in emphysema models [57]) reversed PAH by inhibiting PAEC proliferation (although the authors did 
a)

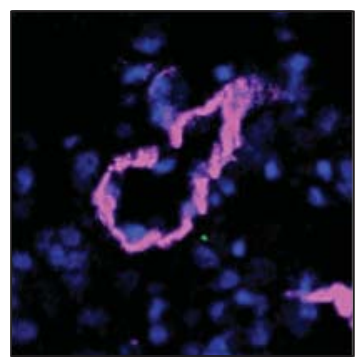

MCT-PAH

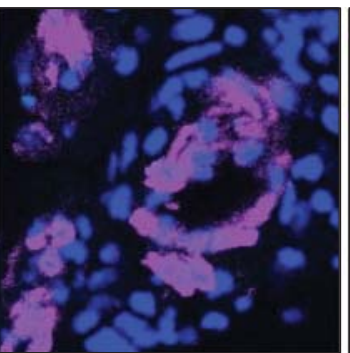

MCT-PAH+PF-228

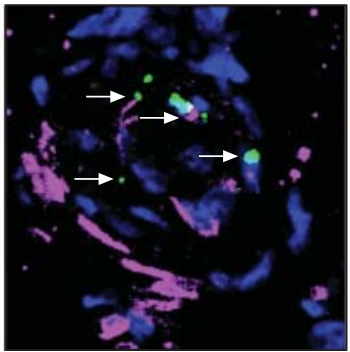

MCT-PAH+SISCR

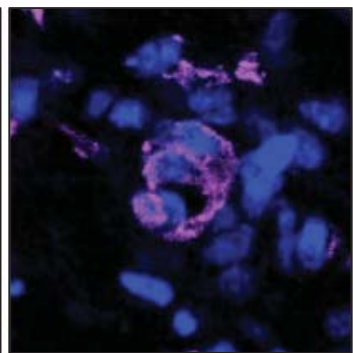

MCT-PAH+SiFAK

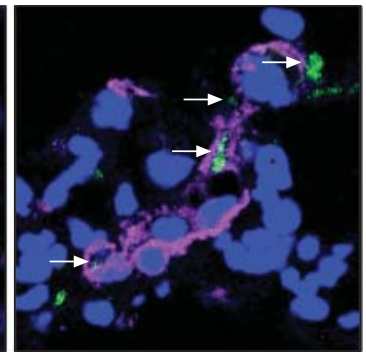

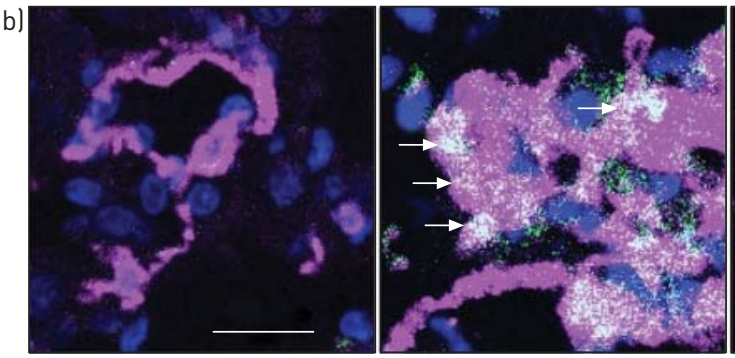
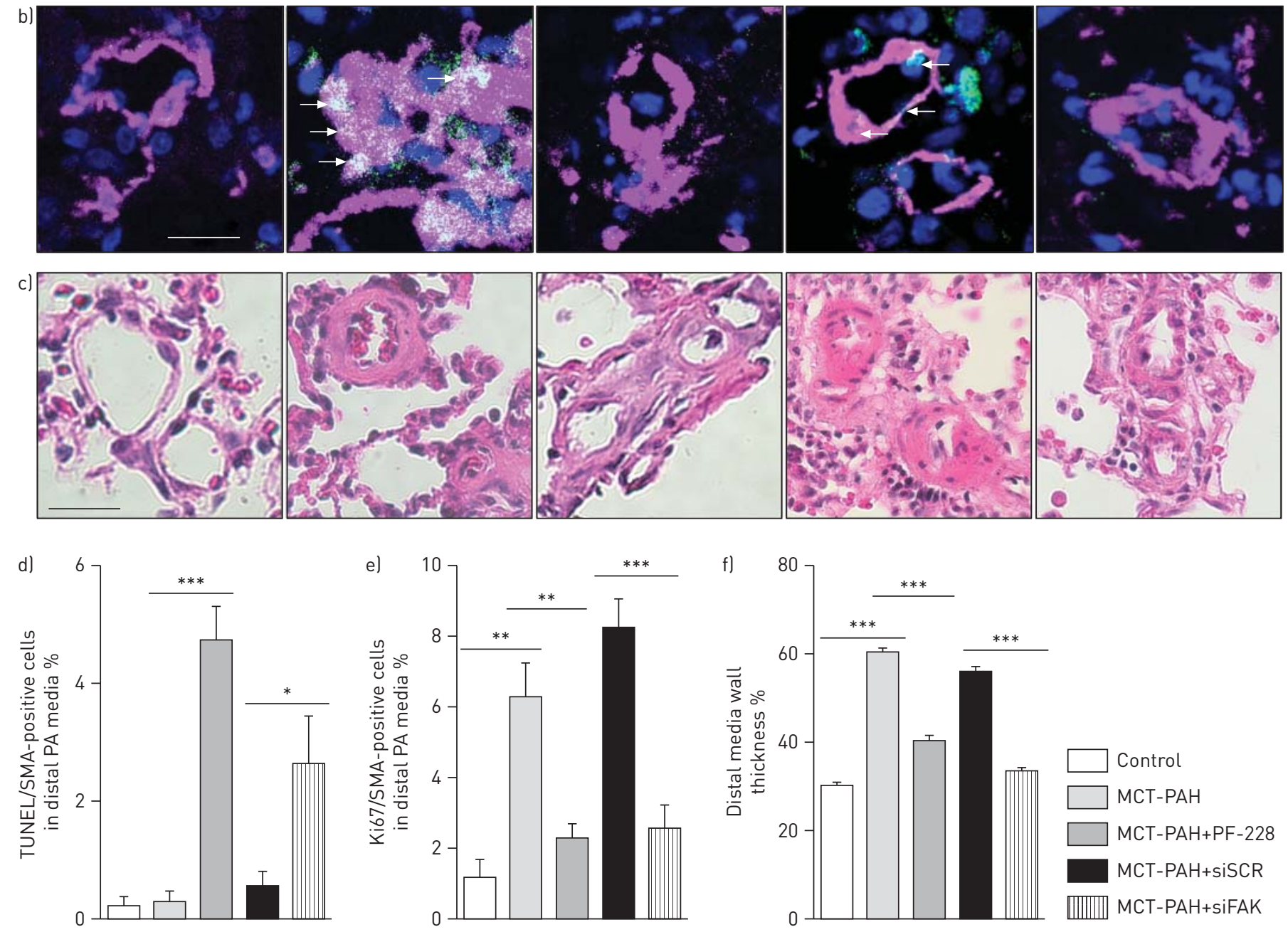

Control

MCT-PAH

MCT-PAH+PF-228

MCT-PAH+siSCR

MCT-PAH+siFAK

FIGURE 7 a, b, d, and e) Focal adhesion kinase (FAK) inhibition decreases pulmonary artery smooth muscle cells (PASMCs) proliferation and survival in resistance PAs in vivo. Improvement of pulmonary arterial hypertension (PAH) condition in rats treated with FAK inhibitors is associated with enhanced apoptosis (percentage of terminal deoxynucleotidyl transferase dUTP nick end labelling (TUNEL)-positive cells (green)) and decreased proliferation (percentage Ki67-positive cells (green)). $\mathrm{n}=10-20$ arteries by rats in five rats in each group. ${ }^{*}: \mathrm{p}<0.05 ;{ }^{* *}$ : $\mathrm{p}<0.01 ;{ }^{* *}$ : $\mathrm{p}<0.001$. c) and f) Show FAK inhibition decreases vascular remodelling in distal PAs in vivo. Improvement of PAH condition in rats treated with FAK inhibitors is associated with decreased distal PA wall thickness (H\&E, $\mathrm{n}=5-10 \mathrm{PA} / \mathrm{rat}$ with 5 rats/group).

not directly study FAK in this work). However in our model we showed that FAK inhibition did not affect PAEC apoptosis or proliferation. Because of the significant differences between these models, the role of FAK in PAEC remains unclear and more studies are needed. On the other hand, the beneficial effects of Copper inhibition on another PAH model, further supports the notion that FAK may be an important therapeutic target in PAH.

All potential PAH therapies need to be tested on the RV as this tissue is the most important of all in terms of the morbidity and mortality of PAH [58] and a limitation of our work is that we did not study the effects of 
FAK inhibitors directly on the RV. FAK has been shown to play a role in cardiomyocyte hypertrophy, being activated by either mechanical stress [59-61] (which causes a translocation of FAK in the cardiomyocyte nucleus [62-64]) or agonists like ET-1 and Ang-II [65, 66]. Increased phosphorylation of FAK has also been described in hypertrophied RV of rats after MCT injection [67]. Thus, more work is needed to discover the direct effects of FAK inhibitors on RV function. Certainly, our data show that the effects of FAK inhibitors on the RV (even when delivered systemically) were not detrimental overall, as they appear to improve the exercise tolerance of the treated animals, a strong sign of an increase in the cardiac output.

The important work by Mizuno at el. [57], showing that Copper deficiency and the resulting FAK inhibition may promote emphysema, need to be taken into careful consideration in further translational work in PAH with this class of therapies. Again, the fact that our treated animals exercised better after treatment of FAK inhibitors, suggests that there were no significant effects on their lung function and gas exchange, at least during this (admittedly short) treatment period. Our guarded optimism for the translational development of FAK inhibitors is supported by the fact that several of them are now under early phase clinical trials for cancer [68].

\section{Acknowledgements}

We want to thank the Societé Québecoise d'Hypertension Artérielle (SQHA), the Canadian Institute for Health Research (CIHR) for scholarships and fellowships attributed to JM and RP. RP is also awarded by an Alberta Innovates Health Solutions (AIHS) fellowship. Thank you to the tissue bank from the "Centre de Recherche de l'Institut Universitaire de Cardiologie et de Pneumologie de Quebec" for tissue samples.

\section{References}

1 McMurtry MS, Archer SL, Altieri DC, et al. Gene therapy targeting survivin selectively induces pulmonary vascular apoptosis and reverses pulmonary arterial hypertension. J Clin Invest 2005; 115: 1479-1491.

2 Voelkel NF, Cool C, Lee SD, et al. Primary pulmonary hypertension between inflammation and cancer. Chest 1998; 114: 225S-230S.

Paulin R, Courboulin A, Barrier M, et al. From oncoproteins/tumor suppressors to microRNAs, the newest therapeutic targets for pulmonary arterial hypertension. J Mol Med (Berl) 2011; 89: 1089-1101.

4 Zhang S, Fantozzi I, Tigno DD, et al. Bone morphogenetic proteins induce apoptosis in human pulmonary vascular smooth muscle cells. Am J Physiol Lung Cell Mol Physiol 2003; 285: L740-L754.

5 Nohe A, Hassel S, Ehrlich M, et al. The mode of bone morphogenetic protein (BMP) receptor oligomerization determines different BMP-2 signaling pathways. J Biol Chem 2002; 277: 5330-5338.

6 Yang X, Long L, Southwood M, et al. Dysfunctional Smad signaling contributes to abnormal smooth muscle cell proliferation in familial pulmonary arterial hypertension. Circ Res 2005; 96: 1053-1063.

7 Moreno-Vinasco L, Gomberg-Maitland M, Maitland ML, et al. Genomic assessment of a multikinase inhibitor, sorafenib, in a rodent model of pulmonary hypertension. Physiol Genomics 2008; 33: 278-291.

8 Mizuno S, Bogaard HJ, Kraskauskas D, et al. p53 gene deficiency promotes hypoxia-induced pulmonary hypertension and vascular remodeling in mice. Am J Physiol Lung Cell Mol Physiol 2011; 300: L753-L761.

9 Chen SJ, Wang YB, Chen O, et al. [Effect of p21 gene transfection mediated by replication deficient adenovirus on the pulmonary hypertensive rat model]. Zhonghua Er Ke Za Zhi 2008; 46: 139-142.

10 Fouty BW, Grimison B, Fagan KA, et al. p27(Kip1) is important in modulating pulmonary artery smooth muscle cell proliferation. Am J Respir Cell Mol Biol 2001; 25: 652-658.

11 Yu L, Quinn DA, Garg HG, et al. Cyclin-dependent kinase inhibitor p27Kip1, but not p21WAF1/Cip1, is required for inhibition of hypoxia-induced pulmonary hypertension and remodeling by heparin in mice. Circ Res 2005; 97: 937-945.

12 Ravi Y, Selvendiran K, Meduru S, et al. Dysregulation of PTEN in cardiopulmonary vascular remodeling induced by pulmonary hypertension. Cell Biochem Biophys 2011 [In press DOI: 10.1007/s12013-011-9332-z].

13 Paulin R, Courboulin A, Meloche J, et al. Signal transducers and activators of transcription-3/pim1 axis plays a critical role in the pathogenesis of human pulmonary arterial hypertension. Circulation 2011; 123: 1205-1215.

14 Courboulin A, Paulin R, Giguere NJ, et al. Role for miR-204 in human pulmonary arterial hypertension. J Exp Med 2011; 208: 535-548.

15 Paulin R, Meloche J, Jacob MH, et al. Dehydroepiandrosterone inhibits the Src/STAT3 constitutive activation in pulmonary arterial hypertension. Am J Physiol Heart Circ Physiol 2011; 301: H1798-H1809.

16 Bonnet S, Rochefort G, Sutendra G, et al. The nuclear factor of activated T cells in pulmonary arterial hypertension can be therapeutically targeted. Proc Natl Acad Sci USA 2007; 104: 11418-11423.

17 Mitra SK, Schlaepfer DD. Integrin-regulated FAK-Src signaling in normal and cancer cells. Curr Opin Cell Biol 2006; 18: 516-523.

18 Sieg DJ, Hauck CR, Ilic D, et al. FAK integrates growth-factor and integrin signals to promote cell migration. Nat Cell Biol 2000; 2: 249-256.

19 Schlaepfer DD, Hou S, Lim ST, et al. Tumor necrosis factor- $\alpha$ stimulates focal adhesion kinase activity required for mitogen-activated kinase-associated interleukin 6 expression. J Biol Chem 2007; 282: 17450-17459.

20 Rozengurt E. Mitogenic signaling pathways induced by G protein-coupled receptors. J Cell Physiol 2007; 213: $589-602$.

21 Schaller MD. Biochemical signals and biological responses elicited by the focal adhesion kinase. Biochim Biophys Acta 2001; 1540: 1-21.

22 Mitra SK, Hanson DA, Schlaepfer DD. Focal adhesion kinase: in command and control of cell motility. Nat Rev Mol Cell Biol 2005; 6: 56-68.

23 Lietha D, Cai X, Ceccarelli DF, et al. Structural basis for the autoinhibition of focal adhesion kinase. Cell 2007; 129: $1177-1187$. 
24 Schlaepfer DD, Hanks SK, Hunter T, et al. Integrin-mediated signal transduction linked to Ras pathway by GRB2 binding to focal adhesion kinase. Nature 1994; 372: 786-791.

25 Xie B, Zhao J, Kitagawa M, et al. Focal adhesion kinase activates Stat1 in integrin-mediated cell migration and adhesion. J Biol Chem 2001; 276: 19512-19523.

26 Harte MT, Hildebrand JD, Burnham MR, et al. p130Cas, a substrate associated with v-Src and v-Crk, localizes to focal adhesions and binds to focal adhesion kinase. J Biol Chem 1996; 271: 13649-13655.

27 Bellis SL, Miller JT, Turner CE. Characterization of tyrosine phosphorylation of paxillin in vitro by focal adhesion kinase. J Biol Chem 1995; 270: 17437-17441.

28 Morrell NW, Adnot S, Archer SL, et al. Cellular and molecular basis of pulmonary arterial hypertension. J Am Coll Cardiol 2009; 54: S20-S31.

29 Schultze A, Fiedler W Therapeutic potential, limitations of new FAK inhibitors in the treatment of cancer. Expert Opin Investig Drugs. 2010; 19: 777-788.

30 National Institutes of Health. Principles of Laboratory Animal Care. Publication No. 85-23, revised 1996. Bethesda, MD, 1996.

31 World Medical Association Declaration of Helsinki. Recommendations guiding physicians in biomedical research involving human subjects. Cardiovasc Res 1997: 35: 2-3.

32 Bonnet S, Archer SL, Allalunis-Turner J, et al. A mitochondria-K+ channel axis is suppressed in cancer and its normalization promotes apoptosis and inhibits cancer growth. Cancer Cell 2007; 11: 37-51.

33 Calalb MB, Polte TR, Hanks SK. Tyrosine phosphorylation of focal adhesion kinase at sites in the catalytic domain regulates kinase activity: a role for Src family kinases. Mol Cell Biol 1995; 15: 954-963.

34 Slack-Davis JK, Martin KH, Tilghman RW, et al. Cellular characterization of a novel focal adhesion kinase inhibitor. J Biol Chem 2007; 282: 14845-14852.

35 Mukhopadhyay UK, Mooney P, Jia L, et al. Doubles game: Src-Stat3 versus p53-PTEN in cellular migration and invasion. Mol Cell Biol 2010; 30: 4980-4995.

36 Gao H, Priebe W, Glod J, et al. Activation of signal transducers and activators of transcription 3 and focal adhesion kinase by stromal cell-derived factor 1 is required for migration of human mesenchymal stem cells in response to tumor cell-conditioned medium. Stem Cells 2009; 27: 857-865.

37 Geiger B, Bershadsky A. Assembly and mechanosensory function of focal contacts. Curr Opin Cell Biol 2001; 13: 584-592.

38 Schaller MD. Paxillin: a focal adhesion-associated adaptor protein. Oncogene 2001; 20: 6459-6472.

39 Taylor JM, Mack CP, Nolan K, et al. Selective expression of an endogenous inhibitor of FAK regulates proliferation and migration of vascular smooth muscle cells. Mol Cell Biol 2001; 21: 1565-1572.

40 Bond M, Sala-Newby GB, Newby AC. Focal adhesion kinase (FAK)-dependent regulation of S-phase kinaseassociated protein-2 (Skp-2) stability. A novel mechanism regulating smooth muscle cell proliferation. J Biol Chem 2004; 279: 37304-37310.

41 Umesh A, Paudel O, Cao YN, et al. Alteration of pulmonary artery integrin levels in chronic hypoxia and monocrotaline-induced pulmonary hypertension. J Vasc Res 2011; 48: 525-537.

42 Dauer DJ, Ferraro B, Song L, et al. Stat3 regulates genes common to both wound healing and cancer. Oncogene 2005; 24: 3397-3408.

43 Verma NK, Dourlat J, Davies AM, et al. STAT3-stathmin interactions control microtubule dynamics in migrating T-cells. J Biol Chem 2009; 284: 12349-12362.

$44 \mathrm{Ng}$ DC, Lin BH, Lim CP, et al. Stat3 regulates microtubules by antagonizing the depolymerization activity of stathmin. J Cell Biol 2006; 172: 245-257.

45 Gao SP, Bromberg JF. Touched and moved by STAT3. Sci STKE 2006; 2006: pe30.

46 Silver DL, Naora H, Liu J, et al. Activated signal transducer and activator of transcription (STAT) 3: localization in focal adhesions and function in ovarian cancer cell motility. Cancer Res 2004; 64: 3550-3558.

47 Ahn S, Park H. XIAP is essential for shear stress-enhanced Tyr-576 phosphorylation of FAK. Biochem Biophys Res Commun 2011; 399: 256-261.

$48 \mathrm{Hu}$ Y, Bock G, Wick G, et al. Activation of PDGF receptor $\alpha$ in vascular smooth muscle cells by mechanical stress. FASEB J 1998; 12: 1135-1142.

49 O'Callaghan CJ, Williams B. Mechanical strain-induced extracellular matrix production by human vascular smooth muscle cells: role of TGF- $\beta 1$. Hypertension 2000; 36: 319-324.

50 Lehoux S, Esposito B, Merval R, et al. Differential regulation of vascular focal adhesion kinase by steady stretch and pulsatility. Circulation 2005; 111: 643-649.

51 Mizuno S, Bogaard HJ, Kraskauskas D, et al. p53 gene deficiency promotes hypoxia-induced pulmonary hypertension and vascular remodeling in mice. Am J Physiol Lung Cell Mol Physiol.

52 Lim ST, Chen XL, Lim Y, et al. Nuclear FAK promotes cell proliferation and survival through FERM-enhanced p53 degradation. Mol Cell 2008; 29: 9-22.

53 Lim ST, Miller NL, Nam JO, et al. Pyk2 inhibition of p53 as an adaptive and intrinsic mechanism facilitating cell proliferation and survival. J Biol Chem 2010; 285: 1743-1753.

54 Cance WG, Golubovskaya VM. Focal adhesion kinase versus p53: apoptosis or survival? Sci Signal 2008; 1: pe22.

55 Sakao S, Taraseviciene-Stewart L, Lee JD, et al. Initial apoptosis is followed by increased proliferation of apoptosisresistant endothelial cells. FASEB J 2005; 19: 1178-1180.

56 Mathew R, Huang J, Shah M, et al. Disruption of endothelial-cell caveolin- $1 \alpha /$ raft scaffolding during development of monocrotaline-induced pulmonary hypertension. Circulation 2004; 110: 1499-1506.

57 Mizuno S, Yasuo M, Bogaard HJ, et al. Copper deficiency induced emphysema is associated with focal adhesion kinase inactivation. PLoS One 2012; 7: e30678.

58 Voelkel NF, Quaife RA, Leinwand LA, et al. Right ventricular function and failure: report of a National Heart, Lung, and Blood Institute Working Group on cellular and molecular mechanisms of right heart failure. Circulation 2006; 114: 1883-1891.

59 Franchini KG, Torsoni AS, Soares PH, et al. Early activation of the multicomponent signaling complex associated with focal adhesion kinase induced by pressure overload in the rat heart. Circ Res 2000; 87: 558-565.

60 Torsoni AS, Constancio SS, Nadruz W Jr, et al. Focal adhesion kinase is activated and mediates the early hypertrophic response to stretch in cardiac myocytes. Circ Res 2003; 93: 140-147. 
61 Torsoni AS, Marin TM, Velloso LA, et al. RhoA/ROCK signaling is critical to FAK activation by cyclic stretch in cardiac myocytes. Am J Physiol Heart Circ Physiol 2005; 289: H1488-H1496.

62 Fonseca PM, Inoue RY, Kobarg CB, et al. Targeting to C-terminal myosin heavy chain may explain mechanotransduction involving focal adhesion kinase in cardiac myocytes. Circ Res 2005; 96: 73-81.

63 Yi XP, Zhou J, Huber L, et al. Nuclear compartmentalization of FAK and FRNK in cardiac myocytes. Am J Physiol Heart Circ Physiol 2006; 290: H2509-H2515.

64 Senyo SE, Koshman YE, Russell B. Stimulus interval, rate and direction differentially regulate phosphorylation for mechanotransduction in neonatal cardiac myocytes. FEBS Lett 2007; 581: 4241-4247.

65 Eble DM, Strait JB, Govindarajan G, et al. Endothelin-induced cardiac myocyte hypertrophy: role for focal adhesion kinase. Am J Physiol Heart Circ Physiol 2000; 278: H1695-H1707.

66 Taylor JM, Rovin JD, Parsons JT. A role for focal adhesion kinase in phenylephrine-induced hypertrophy of rat ventricular cardiomyocytes. J Biol Chem 2000; 275: 19250-19257.

67 Umar S, Hessel M, Steendijk P, et al. Activation of signaling molecules and matrix metalloproteinases in right ventricular myocardium of rats with pulmonary hypertension. Pathol Res Pract 2007; 203: 863-872.

68 Study Of PF-00562271, including patients with pancreatic, head and neck, prostatic neoplasms. http://clinicaltrials. gov/show/NCT00666926 Last accessed: March 14, 2013 Last updated: March 14, 2013. 